\title{
Vein of Galen Malformation with Cerebral Calcification: A Reversible Cause of Neurodegenerative Disease
}

\author{
Stephen J. Phillips, Joseph M. Dooley and Peter R. Camfield
}

\begin{abstract}
This paper reports two children who had mental retardation as the predominant manifestation of vein of Galen malformations. C.T. scans demonstrated extensive intracerebral calcification. Surgery reversed the progressive downhill course in one patient and may have arrested further deterioration in the other. The pathophysiological mechanisms underlying the clinical and radiographic features are discussed.
\end{abstract}

\begin{abstract}
RÉSUMÉ: Malformation de la veine de Galen: une cause inversible de calcification cérébrale et de retard mental progressifs La présente communication rapporte les cas de 2 enfants présentant un retard mental comme manifestation principale de malformations de la veine de Galen. La scanographie cérébrale montrait une calcification cérébrale extensive. La chirurgie a inversé le processus de détérioration chez un patient et a peut-être arrêté sa progression chez l'autre. Les mécanismes pathophysiologiques sous-jacents aux manifestations cliniques et radiologiques sont discutées.
\end{abstract}

Can. J. Neurol. Sci. 1986; 13:103-106

Vein of Galen malformations (VGM) have been postulated to result from fistular communication between diencephalic choroidal vessels and the great cerebral vein during embryogenesis.' In the neonatal period the presentation is determined by the magnitude of the arteriocardiac failure and cranial bruit..$^{2-4}$ Smaller shunts present in infancy, childhood, and adolescence with craniomegaly and cranial bruit, seizures, headaches, exercise syncope, and subarachnoid heamorrhage (SAH). ${ }^{5.6}$ The oldest patient reported in the literature is a 62 year old man who presented with SAH. ${ }^{7}$ Before 1973 the diagnosis was confirmed by cerebral angiography and while this investigation remains a prerequisite for surgical therapy, several reports have shown that VGM are demonstrable by computerized tomographic (CT) scanning. ${ }^{8-13}$

Mental retardation (MR) is a recognized complication of $\mathrm{VGM}^{5}$ which has not been emphaşized in the literature. This paper describes 2 patients in whom MR was the predominant clinical manifestation. Following surgery one improved dramatically; the other was left with a focal deficit but has not suffered further intellectual deterioration. In both, the VGM was demonstrated by CT but on each scan the most striking abnormality was widespread cerebral calcification.

\section{RePORT OF CASES}

Case 1: This patient was the product of an uncomplicated pregnancy. labour and delivery. His early development was normal. At 6 months of age he had a brief generalized seizure. His height, weight, and head circumference were approximately 2 standard deviations above the mean. The examination was otherwise unremarkable. There was no intracranial calcification on skull x-ray. An EEG demonstrated no abnormalities. A CT scan (unenhanced) was interpreted as normal. but in retrospect showed the VGM (Figure 1). His seizures were difficult to control but at 8 months of age his development was normal. At 12 months there was mild gross-motor delay but normal language development.

At 17 months of age he had a prolonged generalized seizure followed by a left Todd's paralysis. On examination he had a high pitched cry. cortical blindness, marked hypotonia, hyperreflexia and a left hemiparesis. His head circumference was normal and there was no evidence of heart failure or cranial bruit. Developmentally he had regressed to a 2-3 month level with the reappearance of primitive reflexes. An unenhanced CT scan showed cortical atrophy and widespread cortical. subcortical and periventricular calcification (Figures $2 \& 3$ ). Cerebral angiography demonstrated a VGM fed predominantly by the posterior circulation. The serum calcium and phosphate were normal. Serum titres for TORCH infections were negative.

At 20 months of age his condition was essentially unchanged with his development still at a 2-3 month level. Through a right parietal-occipital craniotomy the aneurysmally dilated vein of Galen was seen to fill the

From the Departments of Medicine and Pediatrics, Dalhousie University, Halifax, Nova Scotia

This paper was presented at the XX Canadian Congress of Neurological Sciences in Montreal in June 1985.

Received August 13, 1985. Accepted January 28, 1986.

Reprint requests to: Dr. J.M. Dooley, Izaak Walton Killam Hospital for Children. P.O. Box 3070. Halifax, Nova Scotia. Canada B3J 3G9 


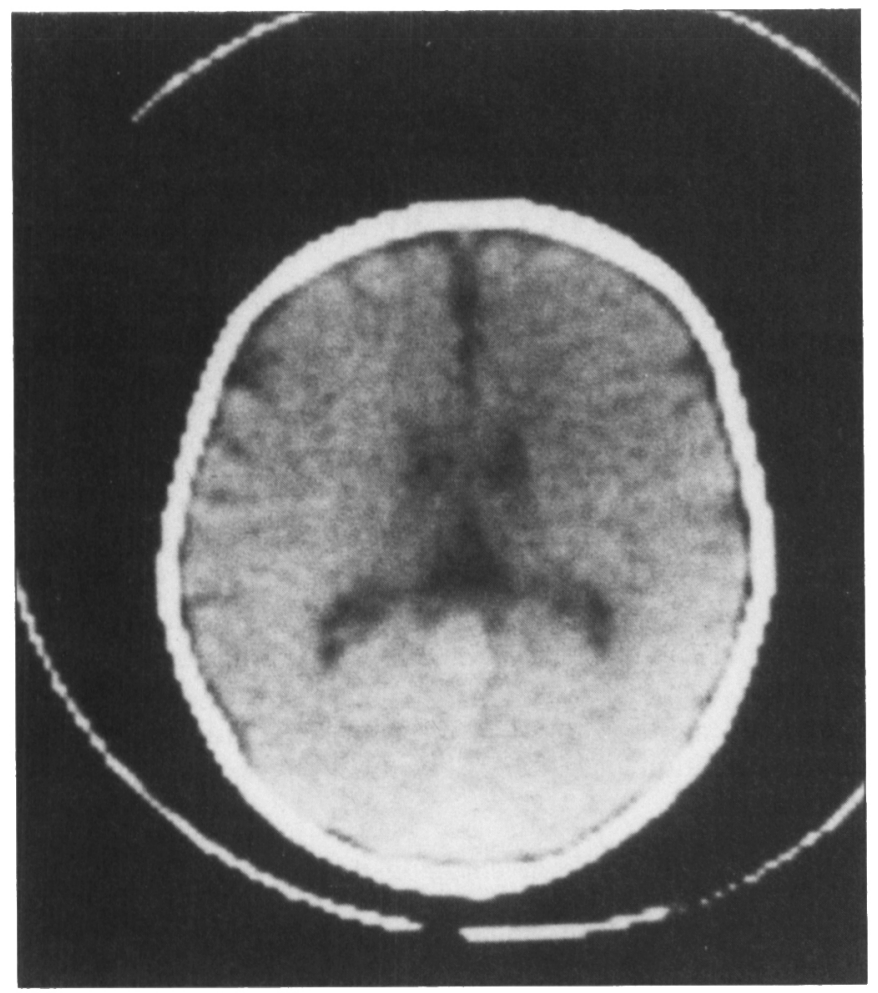

Figure 1: Case 1. Unenhanced CT scan at age 6 months showing lesion in region of splenium of the corpus callosum. No cerebral calcification present.

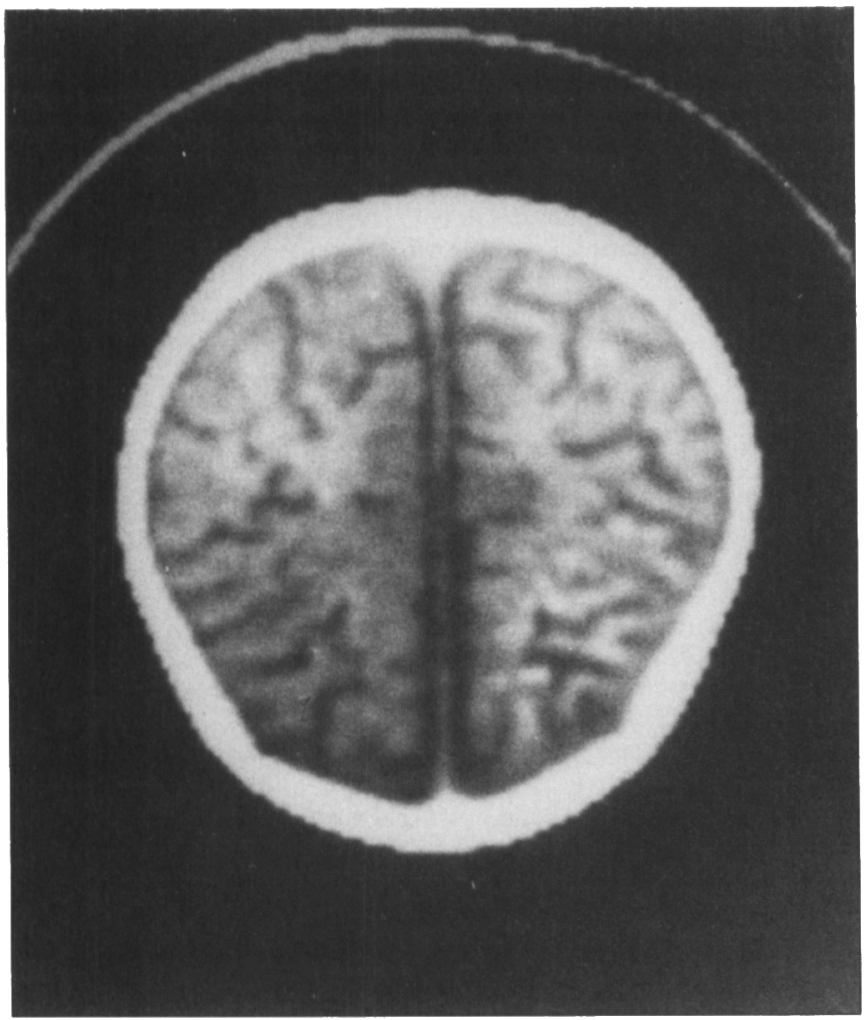

Figure 2: Case 1. Unenhanced CT scan at age 17 months showing atrophy and calcification of the cerebral cortex.

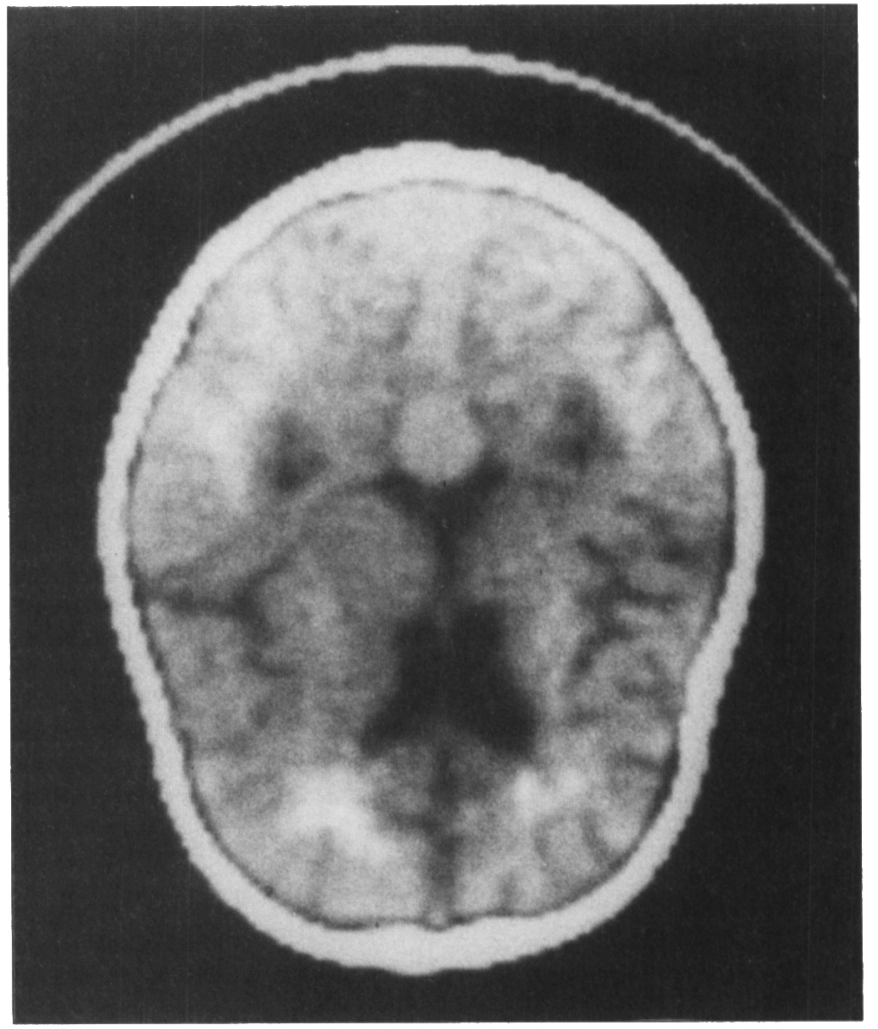

Figure 3: Case 1. Unenhanced CT scan showing a dilated vein of Galen and calcification in the cerebral cortex and subcortical white matter.

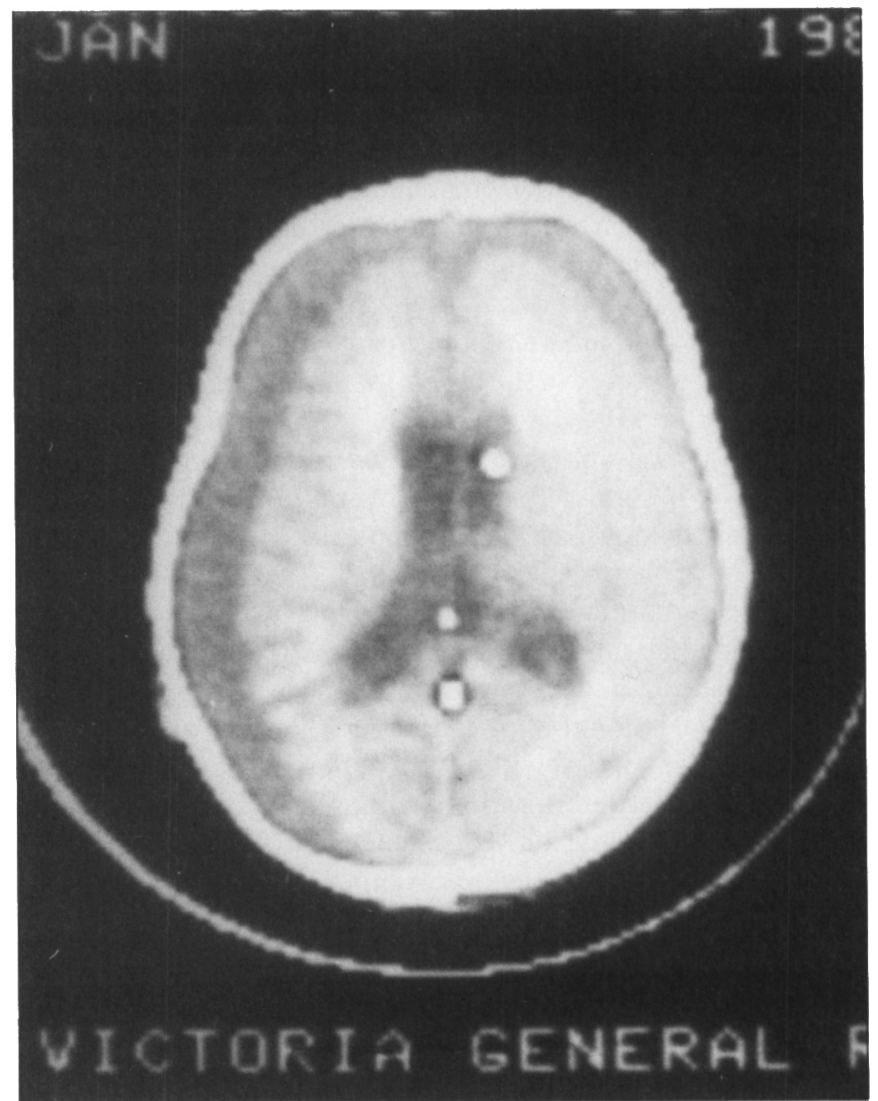

Figure 4: Case 1. Postoperative CT scan (unenhanced) showing bilateral subdural hygromas. 
posterior cisterna ambiens. Several feeding vessels arising from the posterior cerebral and superior cerebellar arteries on both sides were clipped. This resulted in "collapse" of the brain producing a large brain-skull discrepancy (Figure 4) which was visible at the time of surgery. Bilateral subdural-peritoneal shunts were inserted.

Ten months after surgery (age 30 months) considerable improvement was evident. The Bayley Scales of Infant Development ${ }^{14}$ indicated that his mental development was at a 12 month level and that his psychomotor development had reached the 7 month level. Vision had returned. He was able to sit unsupported but made no stepping movements. He tended to hold his left upper extremity in a flexed, adducted posture but was able to utilize a raking grasp with the left hand. On the right he demonstrated an immature pincer grasp and could transfer objects from hand to hand. There was increased tone and hyperreflexia in both lower extremities. He used about a dozen words with meaning but was not speaking in sentences. Seizure control had improved. A repeat CT scan still showed extensive cerebral calcification (Figure 5) and almost complete resolution of the subdural hygromas.

Case 2: This boy was born at 36 weeks gestation following an uncomplicated twin pregnancy. He was noticed to have a large head at 4 weeks of age and was admitted to hospital at 6 months. His head circumference was greater than 2 standard deviations above the mean and his motor skills were delayed. The remainder of his examination was unremarkable. There was minimal cardiac enlargement on chest $\mathrm{x}$-ray and a skull radiograph showed separation of the sutures but no intracranial calcification. Air ventriculography demonstrated moderate enlargement of the lateral and third ventricles but the aqueduct and fourth ventricle were normal in size, position, and shape. A ventriculoperitoneal shunt was inserted. Seizures began in the postoperative period.

At 4 years of age a CT scan showed extensive calcification throughout both cerebral hemispheres. Psychological testing at age 6 years revealed a developmental quotient of 75 . His seizures were difficult to control with anticonvulsant drugs and repeated electroencephalograms showed multifocal epileptiform abnormalities.

Cerebral angiography at age 7 revealed a large VGM (Figure 6). The preferential flow from all four major cerebral vessels was to the malformation. A CT scan showed extensive cortical and deep hemispheric

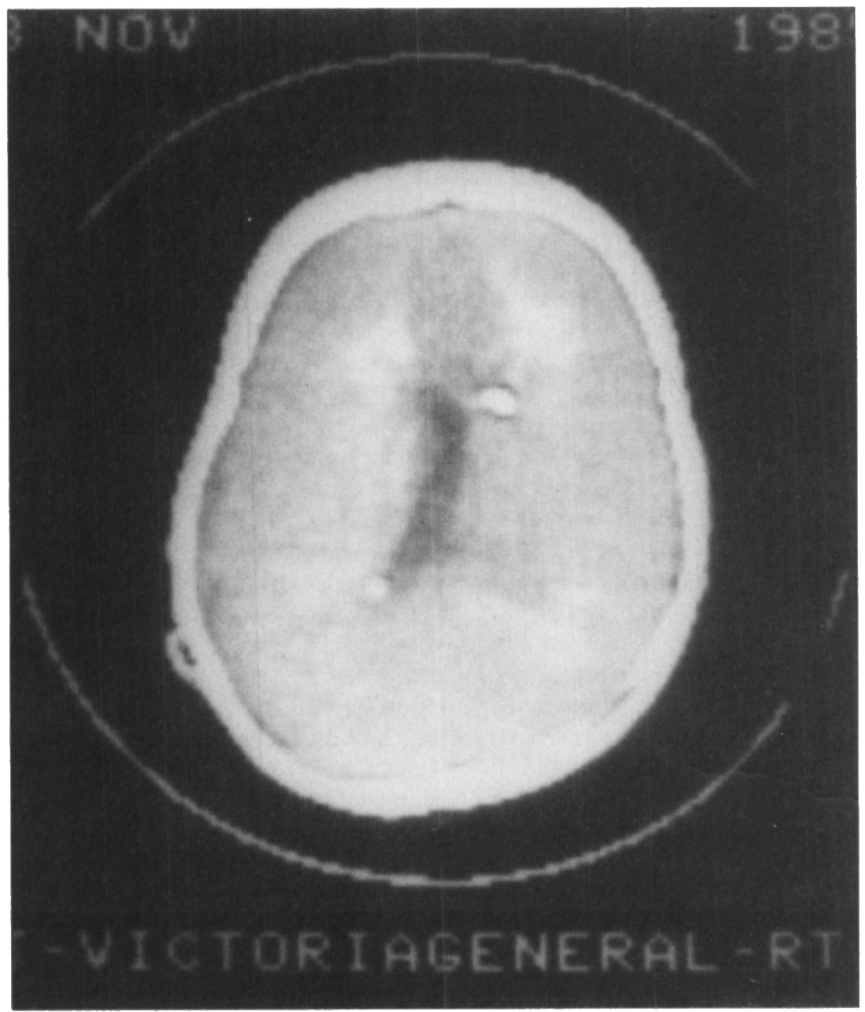

Figure 5: Case 1. Unenhanced CT scan at age 30 months. calcification (Figure 7). The VGM appeared as a tear-drop shaped density in the region of the splenium of the corpus callosum (Figure 8 ). Aphasia and a right hemiparesis complicated surgical clipping of the arterial feeders.

At 11 years, he was moderately mentally retarded and had a left exotropia, left homonymous hemianopia, bilateral optic atrophy and generalized hypertonia. more marked on the right. His intellectual deficit has not progressed.

\section{Discussion}

The loss of intellectual abilities which dominated the clinical picture in these two patients has not been previously emphasized as a major manifestation of VGM. Patient I was developmentally normal when he was examined at 6 months of age because of seizures. By 17 months he had regressed to a 3 month level with the reappearance of primitive reflexes. This marked deterioration was clearly reversed by surgery. Although still retarded at the age of $2 \frac{1}{2}$ years ( 10 months post-surgery) his development seemed to be merely delayed rather than deviated from normal. Patient 2 showed mild developmental delay at 6 months of age when evaluated for progressive macrocephaly. Air ventriculography failed to demonstrate a cause for his hydrocephalus, so it is possible that this was due to increased intracranial venous pressure. ${ }^{15}$ Surgery was complicated by the appearance of new focal signs and he is moderately mentally retarded but his deficits have not progressed. Thus, VGM should be considered in the differential diagnosis of infants with suspected neurodegenerative disease, especially if intracranial calcification is present.

Periventricular calcification has been reported in neonates dying from heart failure secondary to VGM. ${ }^{16.17}$ White matter calcification has also been demonstrated in a mentally normal 20 month old child with a VGM. ${ }^{13}$ Cortical calcification with underlying atrophy has been documented in a 2 year old with a posterior fossa dural arteriovenous malformation ${ }^{18}$ but no clini-

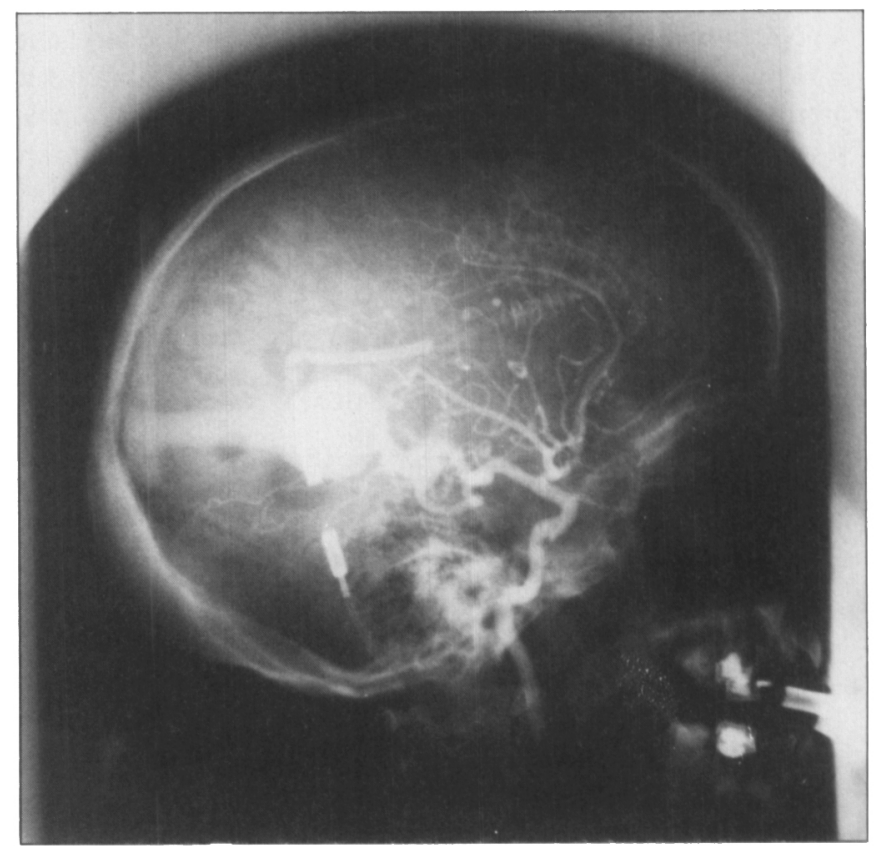

Figure 6: Case 2. Left carotid angiogram. Multiple vessels maimly arising from the posterior cerebral artery feed into a saccular vein of Galen which in turn drains into a dilated straight sinus. 


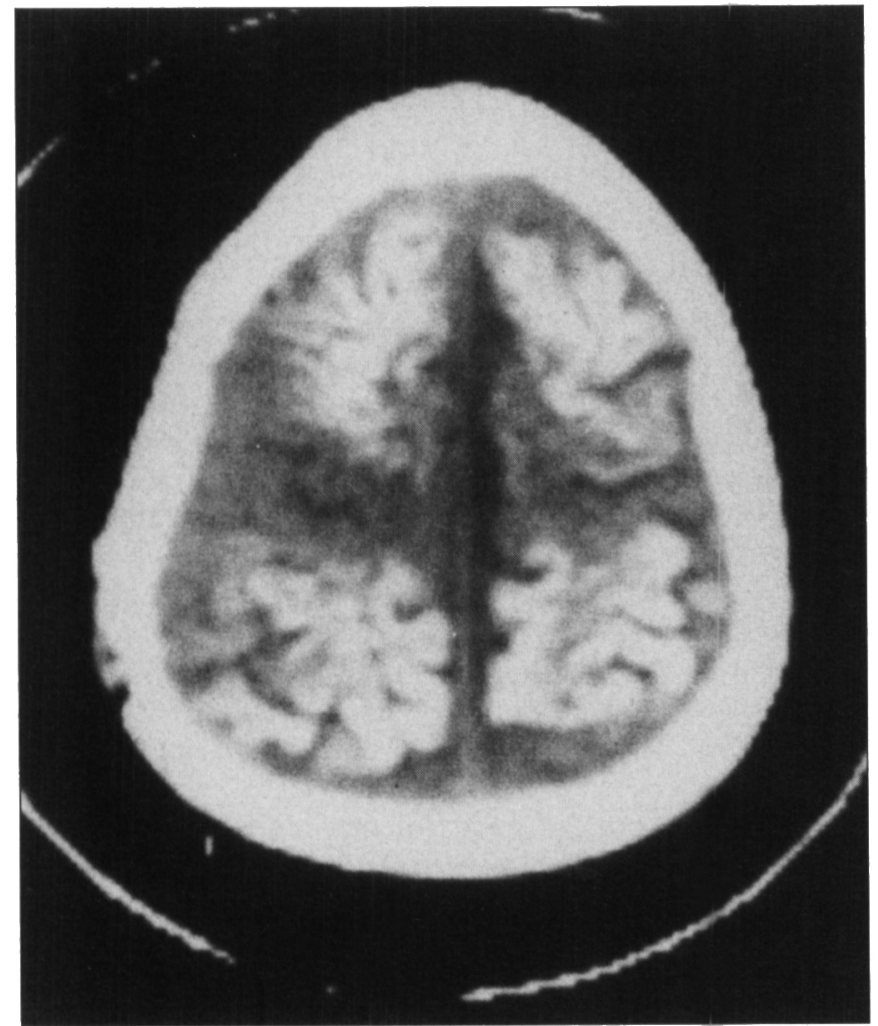

Figure 7: Case 2. Unenhanced CT scan showing atrophy and calcification of the cerebral cortex.

cal details were given. The two patients described here had extensive cerebral calcification. The improvement seen in case 1 despite persisting radiographic abnormalities suggest that calcium deposition per se is not the sole explanation for the clinical features.

In case I, the intra-operative observation of brain collapse was presumably due to a marked fall in cerebral blood volume. It is postulated that prior to surgery the arteriovenous fistula produced venous hypertension and an expanded blood volume. It is also possible that preferential flow of arterial blood to the VGM rendered much of the brain chronically hypoperfused via a steal phenomenon. The exact pathogenesis of the cerebral dysfunction and calcification in these cases is unclear, however, early intervention is important to prevent further neurological deterioration.

\section{ACKNOWLEDGEMENTS}

The authors are grateful to Dr. W. Howes F.R.C.S.(C) for his helpful comments. Dr. J. Byrne Ph.D performed the psychological testing. SJP is a Weston Stroke Fellow.

\section{REFERENCES}

1. Padget DH. The cranial venous system in man in reference to development adult configuration, and relation to the arteries. Am J Anat 1956: 98: 307-355.

2. Gomez MR, Whitten CF, Nolke A et al. Aneurysmal malformation of the great vein of Galen causing heart failure in early infancy. Report of five cases. Pediatrics 1963: 31: 400-441.

3. Stern L. Ramos AD. Wiglesworth FW. Congestive heart failure secondary to cerebral arteriovenous aneurysm in the newborn infant. Am J Dis Child 1968: 155: 581-587.

4. Moodie DS, Sterba R. Rothner AD et al. Great vein of Galen malformations in infancy. Cleve Clin Q 1983: 50: 295-301.

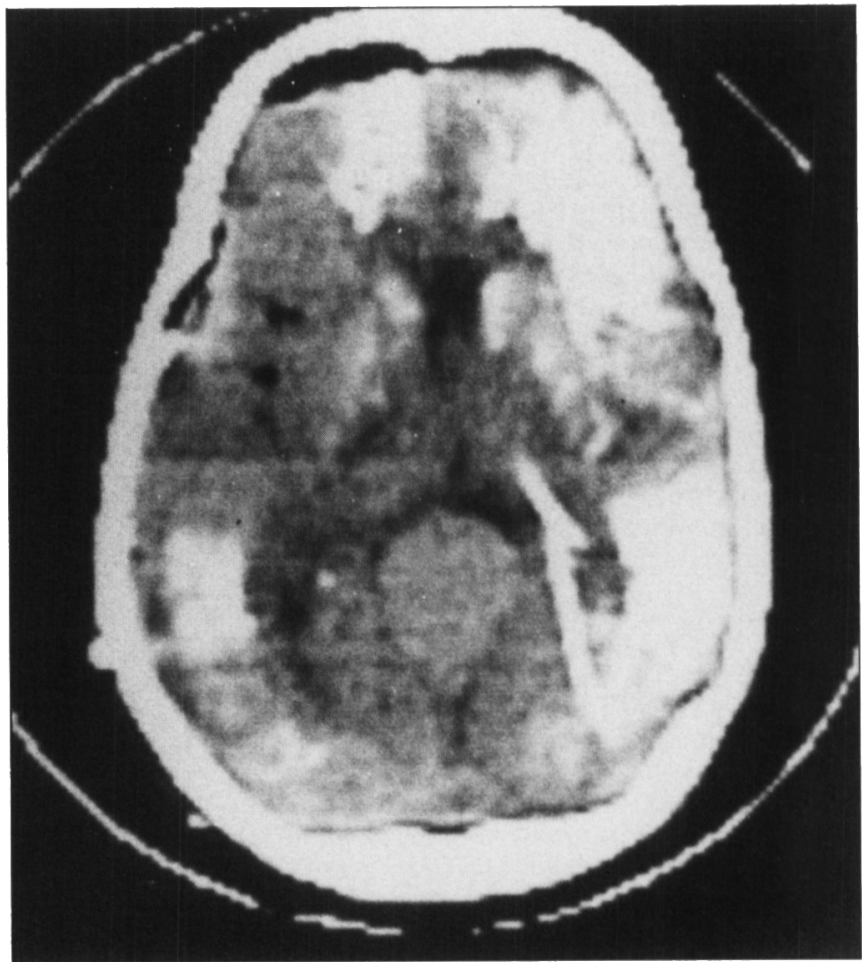

Figure 8: Case 2. Unenhanced CT scan showing the dilated vein of Galen and calcification in the cerebral cortex, subcortical white matter and basal ganglia.

5. Gold AP, Ransohoff J, Carter S. Vein of Galen malformation. Acta Neurol (Scand) 1964: 40 (Suppl 11).

6. Amacher AL, Shillito J. The syndromes and surgical treatment of aneurysms of the great vein of Galen. J. Neurosurg 1973; 39: 89-98.

7. Fernandez RE. Kishore PR, Wilson M et al. Unusual manifestations of vein of Galen aneurysm AJNR 1982: 3: 440-441.

8. MacPherson P, Teadale GM, Lindsay KW. Computed tomography in diagnosis and management of aneurysm of the vein of Galen. J Neurol Neurosurg Psychiatr 1979: 42: 786-789.

9. Spallone A. Computed tomography in aneurysms of the vein of Galen. J Comput Assist Tom 1979; 3(6): 799-782.

10. Iannucci AM, Buonanno F, Rizzuto $\mathrm{N}$ et al. Arteriovenous aneurysm of the vein of Galen: a clinical, angiographic and neuropathological study. J Neurol Sci 1979: 40: 29-37.

11. Martelli A. Scotti G, Harwood-Nash DC et al. Aneurysms of the vein of Galen in children: CT and angiographic correlation. Neuroradiology 1980: 20: 123-133.

12. Rao KC. Chiantella NM, Arora Set al. Intracranial venous aneurysms: vein of Galen and other similar vascular anomalies. CT 1983: 7: 345-350.

13. Diebler C, Dulac O. Renier D et al. Aneurysms of the vein of Galen in infants aged 2-15 months. Diagnosis and natural evolution. Neuroradiology. 1981: 21: 185-197.

14. Bayley N. Bayley scales of infant development. California: The Pyschological Corp. 1969.

15. Rosman NP. Shands KN. Hydrocephalus caused by increased intracranial venous pressure: a clinicopathological study. Ann Neurol 1978: 3: 445-450.

16. Norman MC. Becker LE. Cerebral damage in neonates resulting from arteriovenous malformations of the vein of Galen. J Neurol Neurosurg Psychiatr 1974: 37: 252-258.

17. Perez Fontan J. Herrera M. Fina A. Peguero G. Periventricular calcifications in a newborn associated with aneurysm of the great vein of Galen. Pediatr Radiol 1982: 12: 249-25I.

18. Brunelle FO, Harwood-Nash DC, Fitz CR, Chuang SH. Intracranial vascular malformations in children: computed tomographic and angiographic evaluation. Radiology 1983: 149: 455-461. 